\title{
Extrusion and characterization of aluminum/graphene composites
}

Maik Negendank, Hamidreza Rabi Faezi, Oleksandr Ovsianytskyi, Oliver Goerke, Aleksander Gurlo and Sören Müller

Maik Negendank. Extrusion Research and Development Center of TU Berlin, Gustav-Meyer-Allee 25, 13355 Berlin, Germany.

Corresponding author: Negendank, Maik. E-mail address: maik.negendank@strangpressen.berlin

Hamidreza Rabi Faezi. Extrusion Research and Development Center of TU Berlin, Gustav-Meyer-Allee 25, 13355 Berlin, Germany

Oleksandr Ovsianytskyi. Chair of Advanced Ceramic Materials, Hardenbergstraße 40, 10623 Berlin, Germany

Oliver Goerke. Chair of Advanced Ceramic Materials, Hardenbergstraße 40, 10623 Berlin, Germany

Aleksander Gurlo. Chair of Advanced Ceramic Materials, Hardenbergstraße 40, 10623 Berlin, Germany

Sören Müller. Extrusion Research and Development Center of TU Berlin, Gustav-Meyer-Allee 25, 13355 Berlin, Germany

Abstract. Since its first synthesis in 2004 graphene was characterized intensively and exceptional properties in terms of e.g. mechanical strength, stiffness and electrical as well as thermal conductivity were revealed. These properties make graphene very attractive to be applied as additive in composite materials e.g. to increase strength and conductivity compared to the pure matrix material. In this study graphene nano platelets (GNP) in contents of $0.5 \%, 1.0 \%$ and $1.5 \%$ were added to pure (99.7 \%) aluminum powder and dispersed via EIRICH mixer method. This method is very appealing since homogenous mixtures can be achieved in significantly lower time when compared to e.g. the ball milling process. After subsequent cold compaction the composite materials were extruded with three different extrusion ratios. The influence of GNP content and extrusion ratio on the specific extrusion pressure is characterized as well the resulting rod surface quality, respectively. The effects of GNP content and extrusion ratio on homogeneity of graphene dispersion in the aluminum matrix, the relative density of the composite as well as hardness were also investigated.

Keywords. Aluminum, Graphene Nano Platelets (GNP), Composites, Extrusion, Microstructure, Dispersion, Hardness

\section{Introduction}

Graphene is a new material since its first successful synthesis was achieved in the year 2004 [1]. Since then lots of research was conducted in order to investigate its properties. High mechanical properties like elastic modulus of 1TPa as well as intrinsic strength of $130 \mathrm{GPa}$ were determined [2]. These exceptional values make graphene very attractive to be applied as strengthening additive for composites. In the literature the mechanical properties of $\mathrm{Al} /$ graphene composites have been investigated. For composites that applied aluminum alloys as matrix material, high increases in strength were observed. In case of pure $\mathrm{Al} /$ graphene composites the strength increase was predominantly in the region between $15 \%$ [3] and $85 \%$ [4] and absolute values of yield strength of up to 195 MPa were determined. In order to achieve strong improvements in mechanical strength compared to the monolithic matrix material graphene particles need to be dispersed finely and agglomerations have to be avoided [5]. The aim of the project that this study is a part of, is to investigate methods that should lead to a homogenous distribution of GNPs or carbon-based additives in aluminum MMCs. The presented work in this paper is supposed to generate initial reference data that later can be compared with results of future process investigations and developments.

\section{Experimental}

\subsection{Materials}

For the current investigation gas atomized pure aluminum powder (99.7 \%) was applied as matrix material. The particle size was declared by the manufacturer (TLS Metal Powder company, Germany) to be between 20-63 $\mu$ m, where 
$10 \%$ of particles were lower in size than $28 \mu \mathrm{m}$ and $90 \%$ of Al-powder particles had a diameter below $70 \mu \mathrm{m}$. On the other hand, the applied commercial GNPs were produced by Sigma-Aldrich. The company specified the GNPs with a mean particle size of $6.1 \mu \mathrm{m}$, mean particle thickness of 6-8 $\mathrm{nm}$ and a surface area of $108 \mathrm{~m}^{2} / \mathrm{g}$.

\subsection{Powder processing and billet preparation}

The first step of powder processing was to disperse the GNPs in ethanol for 30 minutes applying an ultrasonic bath in order to separate the GNPs from one another. Subsequently, the GNPs were added to the Al-powder in the mixing pan of the EIRICH mixer. Powder mixtures with graphene contents of $0 \%, 0.5 \%, 1.0 \%$ and $1.5 \%$ (weight percentage) were prepared. Then the powders were mixed for 3 minutes by mechanical stirring with a rotation speed of $40 \mathrm{rpm}$ for the mixer pan and $1800 \mathrm{rpm}$ for the star-shaped mixing tool which spun in the same direction as the mixing pan. The mixed Al/GNP-powders were then filled into a compaction die with a diameter of $29.85 \mathrm{~mm}$. The powder was cold compacted in an uniaxial press by applying a defined force/pressure onto the compaction punch. In a first step the influence of compaction pressure on the resulting density of pure Al-compacts was investigated (see results). Based on the results the compaction pressure was defined to $300 \mathrm{MPa}$. This resulted in compacted tablets of $20 \mathrm{~mm}$ in height and about $35 \mathrm{~g}$ in weight. After cold compaction the tablets were vacuum dried for 16 hours at room temperature. The GNP-dispersions in the Al-matrix were investigated by SEM.

\subsection{Extrusion processing}

The extrusion experiments were conducted on a small vertical $0.5 \mathrm{MN}$ extrusion press with a container diameter of 30 $\mathrm{mm}$ at the Extrusion Research and Development Center TU Berlin in direct extrusion mode. Flat face dies $\left(2 \alpha=180^{\circ}\right)$ with different diameters (and thus extrusion ratios $R$ ) of $12 \mathrm{~mm}(\mathrm{R}=6: 1), 10 \mathrm{~mm}(\mathrm{R}=9: 1)$ and $8 \mathrm{~mm}(\mathrm{R}=14: 1)$ were applied. For the die diameter of $10 \mathrm{~mm}$ an alternative die angle of $2 \alpha=90^{\circ}$ was used in order to investigate its effect on e.g. rod surface quality. The extrusion toolings (dies, dummy blocks) and the compacted tablets were heated up to extrusion temperature of $300{ }^{\circ} \mathrm{C}$ in a chamber furnace. For every extrusion experiment three compacted tablets were inserted into the container, hence the billet length was $60 \mathrm{~mm}$. The extrusions were conducted at a ram velocity of 4 $\mathrm{mm} / \mathrm{s}$. From the measured peak values of total extrusion force $\mathrm{F}_{\text {tot }}$ and the cross-sectional area of the container orifice $\mathrm{A}_{0}$ the specific extrusion pressure $\mathrm{P}_{\mathrm{G}}$ was calculated by applying equation 1 .

$$
P_{G}=\frac{F_{t o t}}{A_{0}}
$$

\subsection{Characterization of extruded rods}

Subsequent to the extrusion experiments the rod surface quality was evaluated. Furthermore, the rods were characterized in terms of microstructure (especially GNP-dispersion), Vickers hardness (HV0.5) as well as density. For microstructural investigations samples were cut from the rods and prepared metallographically. They were then etched with Dix and Keller etchant for $4.5 \mathrm{~min}$ and the microstructure analyzed by optical microscopy as well as SEM. Density of extruded rods was determined by Archimedes' principle through weighting the samples in air and in water environment. 


\section{Results and discussion}

\subsection{Cold uniaxial compaction}

Initially, the pure $\mathrm{Al}$ powder was compacted at room temperature for different compaction pressures. The effect of compaction pressure on the resulting density was investigated. Fig. 1 presents the gained results. It was revealed that the density generally increased with increasing compaction pressure. At a relatively low pressure of 85 Mpa a density of $2.43 \mathrm{~g} / \mathrm{cm}^{3}$ was achieved and applying a pressure of $566 \mathrm{MPa}$ lead to a density of $2.61 \mathrm{~g} / \mathrm{cm}^{3}$. The results also indicated that sufficient stability of the compacts for the handling before and during the extrusion experiments was given at $283 \mathrm{MPa}$ and thus, this pressure was applied for compaction of $\mathrm{Al} / \mathrm{GNP}$ tablets.

The GNP dispersion in the Al/GNP-compacts was investigated by SEM element mapping. Exemplary results are given in Fig. 2. According to the element maps the amounts of identified GNPs (mapped in yellow) increased with increasing GNP content in the powder mixture from $0.5 \%$ GNPs to $1.5 \%$ GNPs. GNP agglomerations were not observed and the particle distribution seemed to be relatively homogeneous.

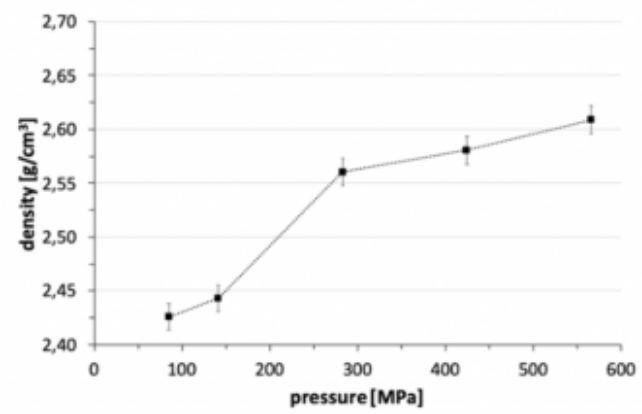

Fig. 1. Effect of compaction pressure on density of pure-Al compacts.

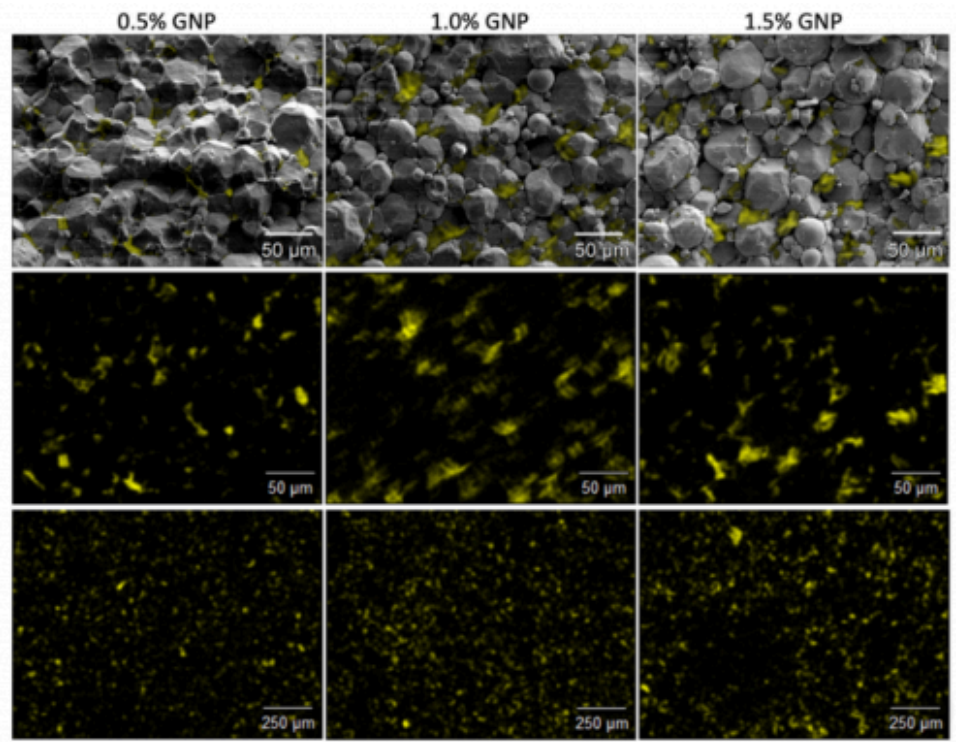


Fig. 2. SEM element mapping of compacts with different GNP-contents (carbon displayed in yellow).

\subsection{Extrusion results}

After the extrusion trials were conducted the specific extrusion pressures were calculated from the measured total extrusion forces according to equation 1. The effect of GNP content and extrusion ratio is given exemplarily for extrusions of pure $\mathrm{Al}$ as well as for $1.5 \%$ GNP content in Fig. 3a. According to that $\mathrm{P}_{\mathrm{G}}$ slightly increases with increasing extrusion ratio for both materials. Furthermore, compared to pure aluminum the specific extrusion pressure at same extrusion ratio is higher for the billet material containing $1.5 \%$ GNPs. Hence, since higher extrusion forces and thus specific extrusion pressures are required for extrusion of the GNP containing material, the addition of $1.5 \%$ GNPs seems to have a strengthening effect to the billet material.

Furthermore, the density of rod sections was determined in dependence of GNP content and extrusion ratio. The results are given in Fig. 3b. The highest density was found for the pure-Al rods. With increasing GNP content, a tendency of decreasing density was observed. This seems to be obvious since the density of GNPs is lower than that of aluminum. Thus, by increasing the GNP content, the density of the composites is reduced. The extrusion ratio did not have a significant effect on the rod density.
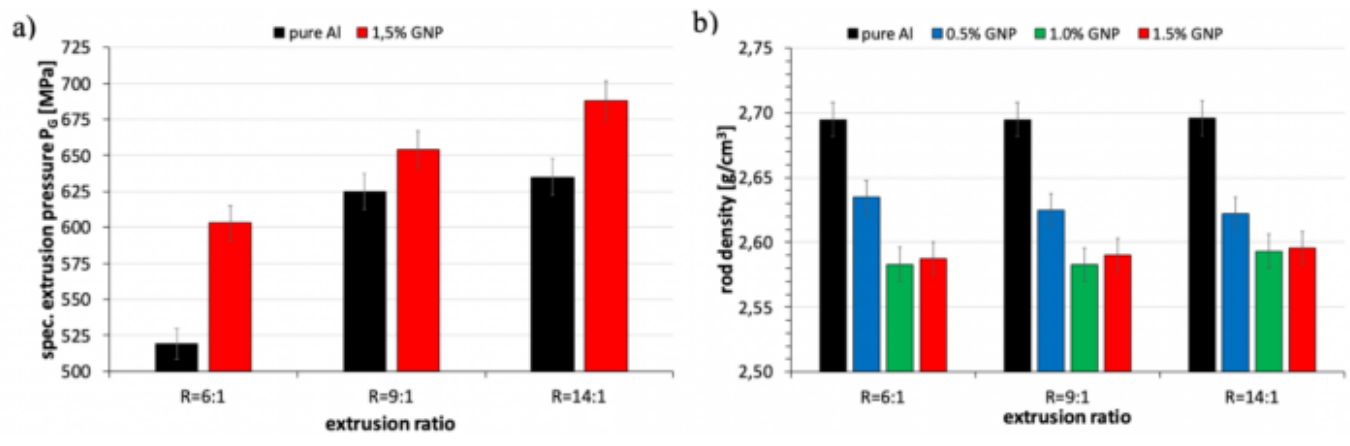

Fig. 3: a) Effect of extrusion ratio and addition of 1.5\% GNPs on specific extrusion pressure $P_{G}$ b) density of extruded rods in dependence of GNP-content and extrusion ratio.

Subsequent to the extrusion experiments the surface quality of the rods was evaluated. Fig. 4 presents the rods extruded through flat face dies (die angle $2 \alpha=180^{\circ}$ ) with die orifice diameters of $12 \mathrm{~mm}$ (extrusion ratio $\mathrm{R}=6: 1$ ) as well as $8 \mathrm{~mm}(\mathrm{R}=14: 1)$. For both extrusion ratios the surface quality in case of pure Al was acceptable, although for the higher extrusion ratio of $\mathrm{R}=14: 1$ some surface marks occurring perpendicular to extrusion direction were observed. They were caused by strong rod vibrations during the extrusion on the vertical extrusion press. As Fig. 4a shows the surface quality decreased drastically when compacts containing GNPs were extruded. The effect increased with increasing GNP content. The surfaces of GNP-containing rods were very rough and even showed cracks. For the higher extrusion ratio of $\mathrm{R}=14: 1$ the surface quality also decreased with increasing GNP content (Fig. $4 \mathrm{~b}$ ) but was slightly better when being compared to those with lower extrusion ratio at same GNP content. 

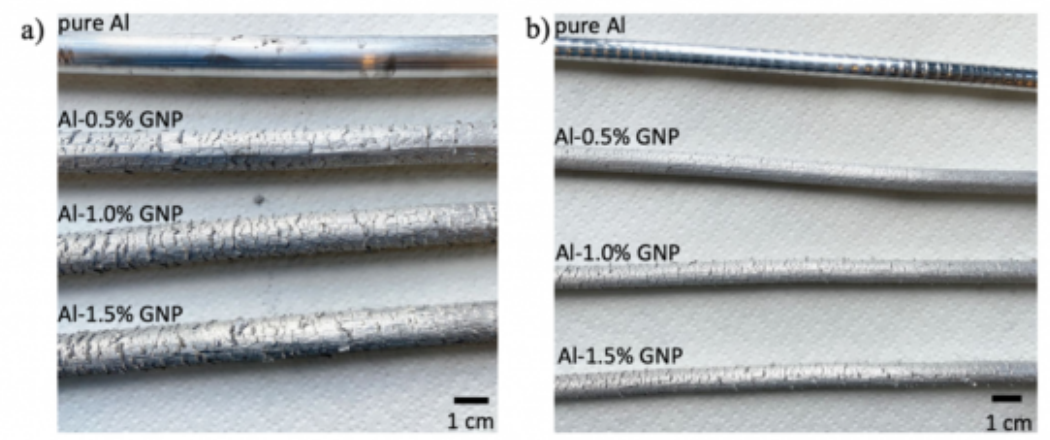

Fig. 4. Influence of GNP content on rod surface quality a) die diameter $12 \mathrm{~mm}(\mathrm{R}=6: 1) \mathrm{b})$ die diameter $8 \mathrm{~mm}(\mathrm{R}=14: 1)$.

In Fig. 5 rods are presented that were extruded through $10 \mathrm{~mm}$ dies with different die angles, where one was a flat face die $\left(2 \alpha=180^{\circ}\right)$ and the other one of conic shape with a die angle of $\left(2 \alpha=90^{\circ}\right)$. The direct comparison reveals that in case of the conic die, a better rod surface quality was achieved especially for GNP contents of $0.5 \%$ and $1.0 \%$. In case of $1.5 \%$ GNPs the flat face die showed a slightly better rod surface since less cracks were observed.

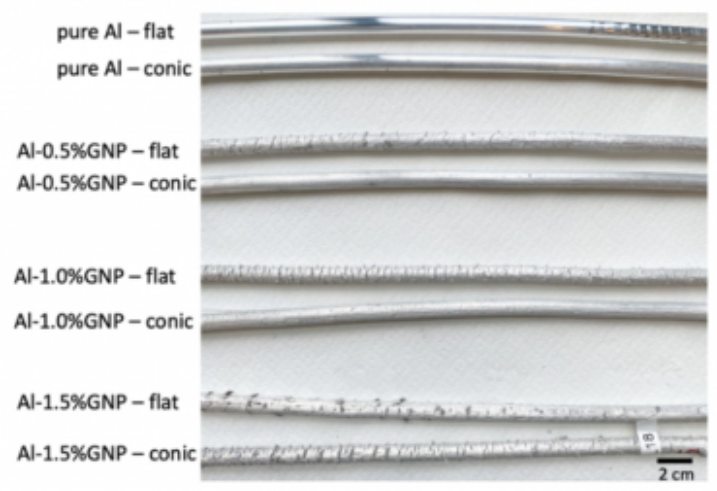

Fig. 5. Effect of die angle $\left(2 \alpha=180^{\circ}\right.$ (flat) and $2 \alpha=90^{\circ}$ (conic) ) on rod surface quality for different GNP contents, rod diameter $10 \mathrm{~mm}(\mathrm{R}=9: 1)$.

\subsection{Microstructure}

After the extrusion trials samples were cut from the rods in order to investigate the GNP distribution at the center and the rim of the rods' cross sections. The results are given in Fig. 6. Fig. 6a displays the GNP distribution at the center and the rim of the rods extruded at $\mathrm{R}=6: 1$ in dependency of GNP content. At the low GNP content of $0.5 \%$ there is no significant difference in the microstructures of center and rim position. With increasing GNP content at the center position a network-like structure was found. It seems like the GNPs increasingly aligned around the Al-grains. GNPs are predominantly of lengthy shape in the image plane as was confirmed by SEM analysis (Fig. 7a). In contrast to that the GNPs seemed to be predominantly round-shaped at the rim positions of the cross sections (Fig. 7b). With increasing GNP content, the mean distance between particles is reduced but no network structure was observed. The microstructural observations might be explained with the differences in strain and strain rate distributions over rod cross section during the extrusion process. The direct extrusion process is characterized with higher strain and strain 
rates on the surface of the extrudates (e.g. rods) compared to the center since the material forming the surface of the extrudate passed the shear intensive zone of the billet and thus, is exposed to high shear stresses [6]. The shear stresses increase for higher die angles [7]. These shear stresses seem to be the reason for the different shape of GNPs at the rim positions. More detailed investigations are necessary to clarify the relationship between metal flow during extrusion and GNP shape and orientation in the extrudates. Furthermore, Fig. 6b presents the microstructures at the center and rim positions at a GNP content of $1 \%$ for increasing extrusion ratios. It can be learned that at the lowest extrusion ratio of $\mathrm{R}=6: 1$ there is a big difference in GNP orientation and shape between center and rim positions. The GNPs were of lengthy shape in the center and formed a network-like structure, while they were more separated and predominantly of round shape at the rim. Though, at the highest extrusion ratio of $\mathrm{R}=14: 1$ these microstructures became more homogeneous and round shaped GNPs were predominant at the rim as well as in the center of the rods. It is assumed that the higher strain and strain rate induced by applying a higher extrusion ratio (and same ram velocity) were the main reasons for the more homogeneous microstructures. Probably, the gradient in strain and strain rate values over the rods' cross sections was not as strong as in case of higher rod diameters (lower extrusion ratios).

The longitudinal section of a $1.5 \%$ GNP sample extruded at R=6:1 (Fig. 7c) shows GNPs aligned behind one another oriented parallelly to the extrusion direction almost in fibrous shape. The displayed section is positioned just below the rod surface. The authors assume that these longitudinal "GNP-fibers" could be the reason for the observed bad surface quality for the GNP containing rods. In direct extrusion the friction conditions in the die bearing between $\mathrm{Al}$ billet material and die material (hot working tool steel H11) are often characterized with high friction coefficients and even sticking friction conditions [7]. So, if high friction forces or even sticking friction occurs between rod surface and the die bearing channel and when furthermore GNPs and Al-matrix are bonded poorly, then shear stresses acting on the rods' surfaces can exceed the intrinsic strength of the Al/GNP-compound and could be responsible for the formation of cracks on the rods' surfaces. This effect would increase for higher GNP-contents since the amount of "GNP-fibers" should increase also and could be the reason for the observed problematic surface quality.
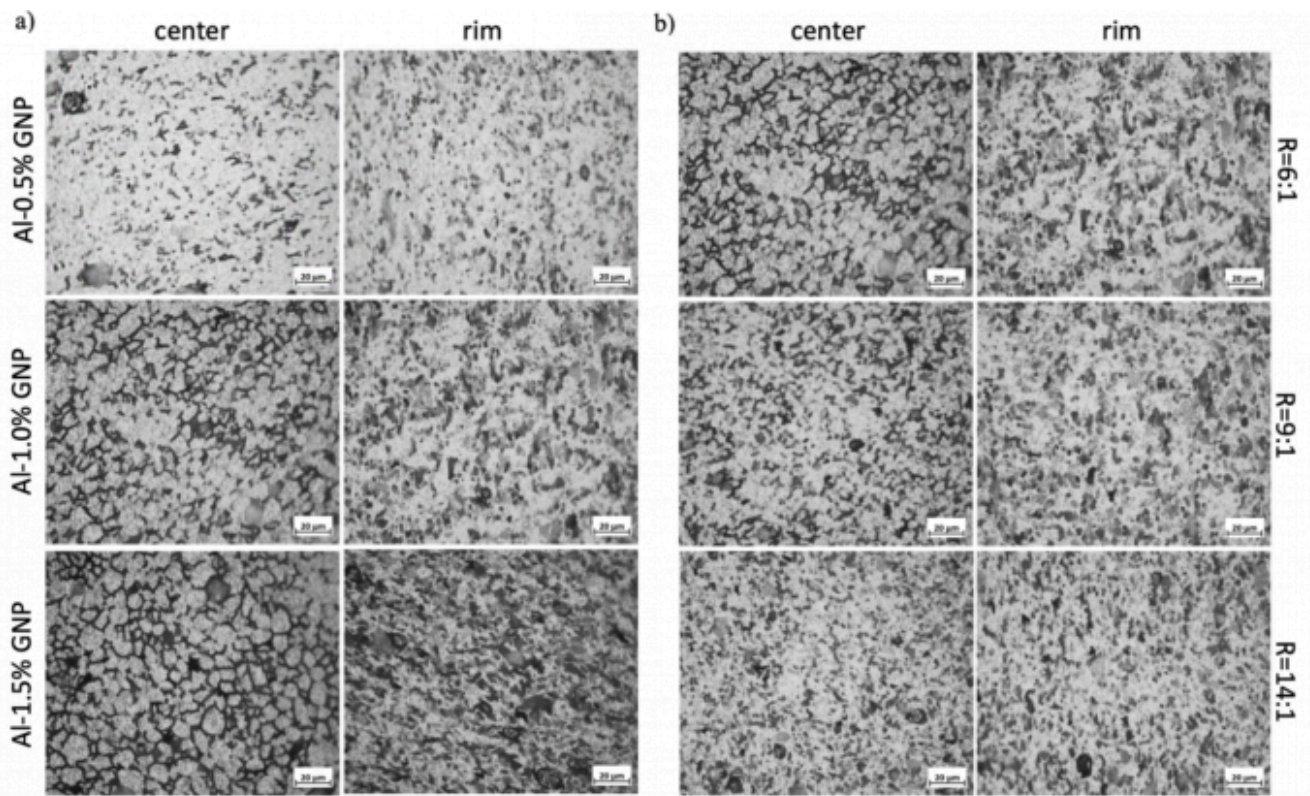

Fig. 6. Microstructure (OM) of rod cross sections at center and rim of rods with a) increasing GNP-content at $\mathrm{R}=6: 1 \mathrm{~b}$ ) increasing extrusion ratio at $1.0 \%$ GNP-content. 


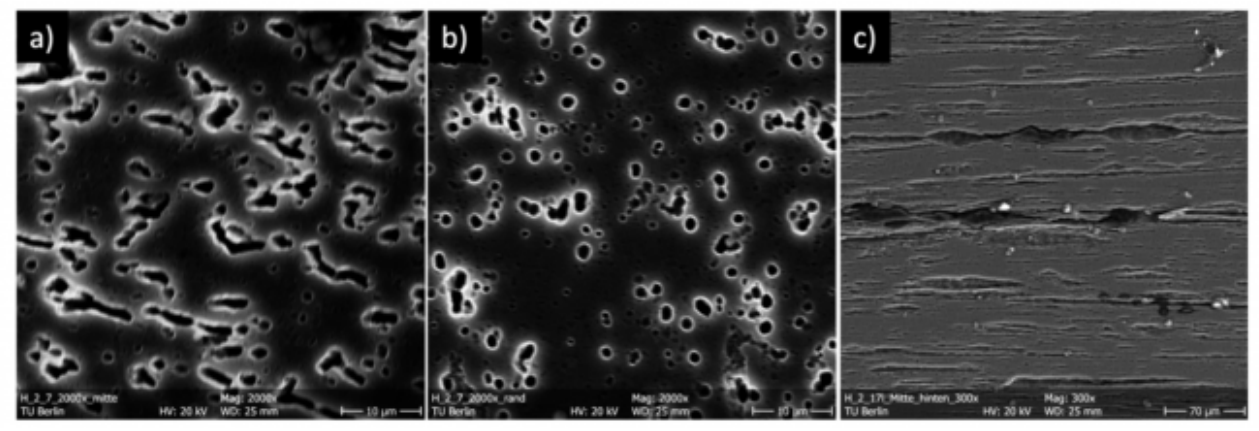

Fig. 7. SEM pictures (cross sections) of GNPs in rod extruded at $\mathrm{R}=6: 1$ with $0.5 \%$ GNPs a) Center b) Rim c) longitudinal section of rod extruded at $\mathrm{R}=6: 1,1.5 \% \mathrm{GNP}$.

\subsection{Hardness}

Vickers hardness was measured at the center and the rim positions of rod samples. The results are presented in Fig. 8. The hardness values at center (Fig. 8a) remained constant around 35-36HV in case of pure Al. The extrusion ratio again did not have an effect for that material in the investigated range. At the extrusion ratios of $R=6: 1$ und $R=9: 1$ the hardness values for the graphene containing materials were either about constant or even reduced compared to pure Al. The strongest reduction of $11 \%$ was found for the sample with 1.5\% GNPs extruded with $\mathrm{R}=9: 1$ via conic die where hardness decreased from 35.5HV to 31.6HV. The only significant effect of the extrusion ratio on Vickers hardness at the center positions was found for the samples containing $1 \%$ GNPs. Here, the hardness increased from 31.6HV (R=6:1) to $36.1 \mathrm{HV}(\mathrm{R}=14: 1)$, which means an increase by $14 \%$.

For the hardness at the rim positions (Fig. 8b) the results were different from those of the center of rods. Here, the hardness was lowest for the pure aluminum samples with values of 35-36HV, which were the same as in the center. Hence, in case of pure Al Vickers hardness was homogenous over the rods' cross sections. But in contrast to results from the center the addition of GNPs to pure Al lead to an increase of hardness values. A tendency of increasing hardness with higher amounts of GNPs was found at the rim positions of extruded rods for a given extrusion ratio. The highest value of $42 \mathrm{HV}$ was measured at $\mathrm{R}=9: 1$ for the highest GNP content of $1.5 \%$. This corresponds to an increase in hardness of about 18\% compared to values of pure aluminum. The extrusion ratio did not have a significant effect on the hardness at the rim position.

Obviously, the observed different hardness values between center and rim positions correspond to the differences in GNP shape or orientation as displayed in Fig. 6 and Fig. 7. According to that, an increase in hardness was achieved when the GNPs are of round shape in the cross sectional area of extruded rods. Additional investigations including TEM analysis are necessary and will be conducted in upcoming investigations in order to determine the effect of GNP orientation on load transfer from Al-matrix to GNPs more detailed. 

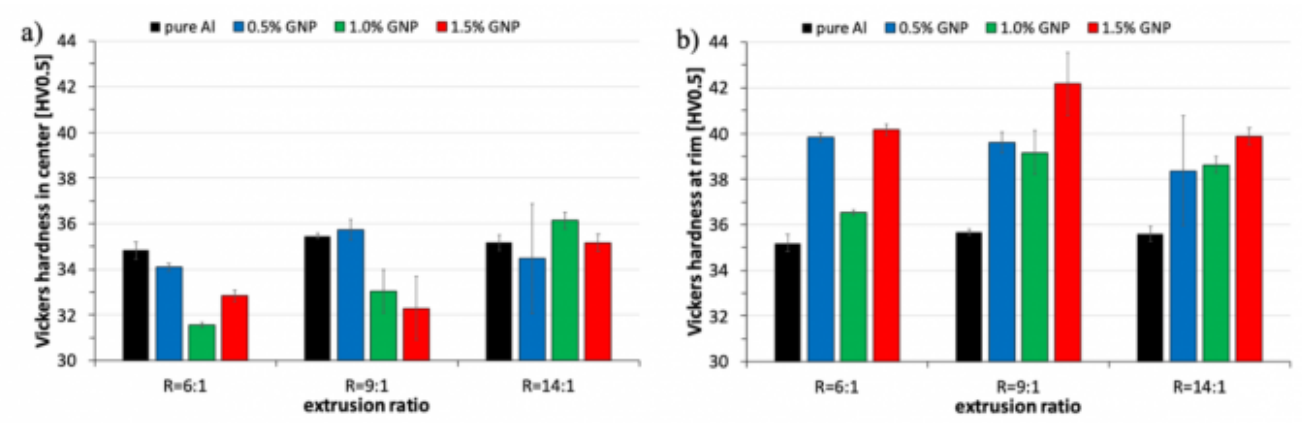

Fig. 8. Vickers hardness in dependence of GNP content and extrusion ratio at a) center of extruded rods b) rim of rods.

\section{Conclusions}

The powder processing, extrusion experiments as well as characterization of extruded rods of Al-GNP composites with GNP contents of $0.5,1.0 \%$ and $1.5 \%$ in comparison to pure $\mathrm{Al}(99.7 \%)$ via same processing route lead to the following conclusions:

- Mechanical stirring of Al/GNP powder mixtures with EIRICH mixer method resulted in homogenous GNP dispersion in compacts and no agglomerations were observed.

- Surface quality of extruded rods decreased with increasing GNP content, but increased for higher extrusion ratios. It was assumed that the formation of fibrous-like GNP-structures below the rod surface lead to reduced strength perpendicular to the extrusion direction. Combined with the high friction conditions between rod surface and die bearing channel during extrusion this could be the reason for the observed surface cracking at high GNP-contents.

- Application of conic die $\left(2 \alpha=90^{\circ}\right)$ showed better rod surface quality than flat face die $\left(2 \alpha=180^{\circ}\right)$. As a reason lower shear stresses in the deformation zone of the conic die are assumed.

- GNPs in the center of rod cross section are oriented differently than on the rim. In the center the GNPs showed a lengthy shape or orientation in the image plane, while they were predominantly round at the rim.

- Increase in hardness at rim positions (up to $+18 \%$ ) was measured. In the center the hardness was constant at best and even reductions of up to $11 \%$ were found. The orientation and shape of GNPs seem to be responsible for the inhomogeneous distribution of harness values along rods' cross sections. Eventually, the GNP shape and orientation affect the load transfer from Al matrix to the GNP particles.

\section{Acknowledgements}

The authors acknowledge the financial support of the German Research Foundation (DFG) for project NE2321/2-1.

\section{Bibliography}

[1] Novoselov KS, Geim AK, Morozov SV, Jiang D, Zhang Y, Dubonos SV, Grigorieva IV et al. Electric field effect in atomically thin carbon films. Science 2004, 306, 666-669.

[2] Lee C, Wei X, Kysar JW, Hone J. Measurement of the Elastic Properties and Intrinsic Strength of Monolayer Graphene. Science 2008, 321, 385-388.

[3] Rashad M, Pan F, Tang A, Asif M. Effect of Graphene Nanoplatelets addition on mechanical properties of pure 
aluminum using a semi-powder method. Progress in Natural Science: Materials International 2014, 24, 101-108.

[4] Bisht A, Srivastava M, Kumar RM, Lahiri I, Lahiri D. Strengthening mechanism in graphene nanoplatelets reinforced aluminum composite fabricated through spark plasma sintering. Materials Science and Engineering: A 2017, 695, $20-28$.

[5] Kumar HP, Xavior MA. Graphene Reinforced Metal Matrix Composite (GRMMC). Procedia Engineering 2014, 97, 1033-1040.

[6] Claves SR, Janiszewska K, Misiolek WZ, Material flow in billets of thin-walled extrusions, Proceedings of the 8th Aluminum Extrusion Technology Seminar and Exposition, 2004, 55-67.

[7] Welo T, Abtahi S, Skauvik I, Storen S, Melander M, Tjotta S. Friction in the bearing channel of aluminium extrusion dies. In: Proceedings of the 15 th Riso International Symposium on Materals Secience: Numerical Predictions of Deformation Processes and the Behaviour of Real Materials, 1994, Anderson SI, Bilde- Sorensen JB, Lorentzen T, Pedersen OB, Sorensen NJ (Eds.), 615-620.

PDF automatically generated on 2021-05-23 12:18:43

Article url: https://popups.uliege.be/esaform21/index.php?id=3714

published by ULiège Library in Open Access under the terms and conditions of the CC-BY License (https://creativecommons.org/licenses/by/4.0) 